\title{
A modular DNA scaffold to study protein-protein interactions at single-molecule resolution
}

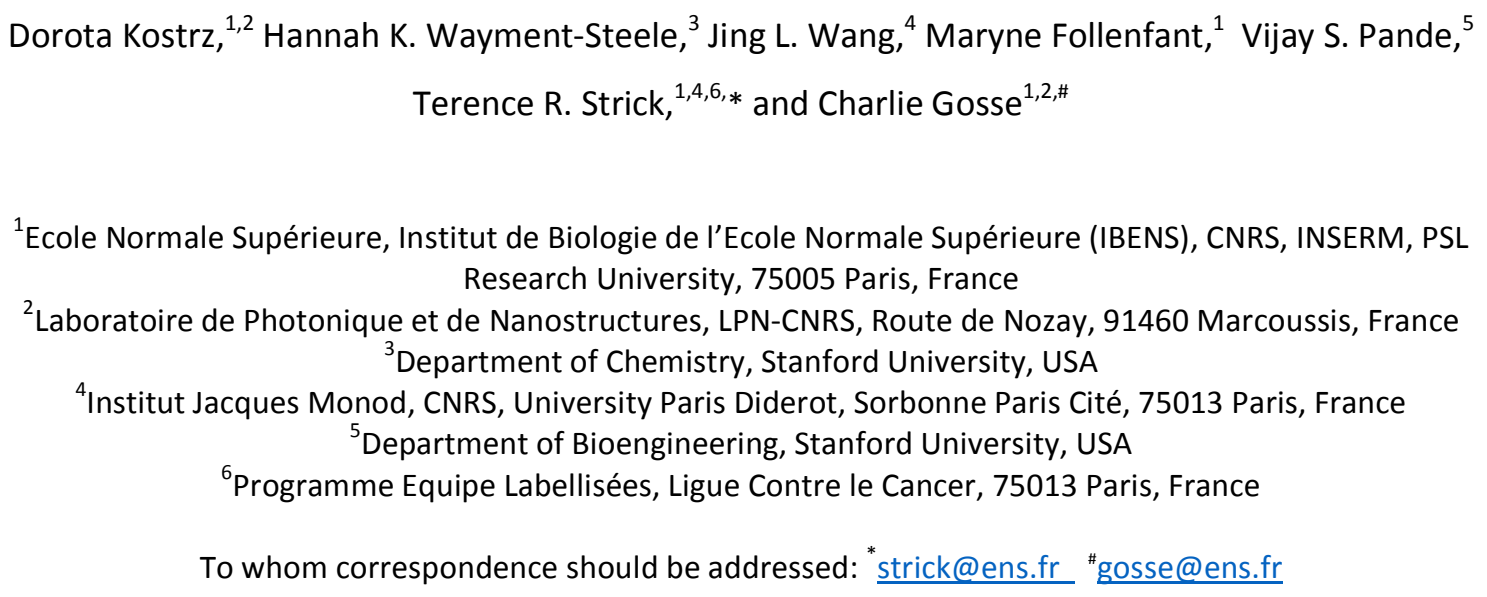

The residence time of a drug on its target has been suggested as a more pertinent metric of therapeutic efficacy than the traditionally used affinity constant. Here we introduce junctured-DNA (JDNA) tweezers as a generic platform which enables real-time observation, at the single-molecule level, of biomolecular interactions. This tool corresponds to a double-strand DNA scaffold which can be nanomanipulated and on which proteins of interest can be engrafted thanks to widely-used genetic tagging strategies. Thus, J-DNA tweezers allow straightforward and robust access to single molecule force spectroscopy in drug discovery, and more generally in biophysics. Proof-of-principle experiments are provided for the rapamycin-mediated association between FKBP12 and FRB, a system relevant in both medicine and chemical biology. Individual interactions were monitored under a range of applied forces and temperatures, yielding after analysis the characteristic features of the energy profile along the dissociation landscape. 
Kinetic characterization of molecular interactions provides essential mechanistic insights into the functioning of biochemical pathways $[1,2,3]$. With increasing frequency it also underpins the initial stages of drug screening and development, as well as later stages of drug refinement [4, 5]. Indeed, two molecules with the same affinity for their target but different binding dynamics may have varied physiological effects in terms of safety and/or efficacy [6]. The most informative measurement strategies currently include biosensors such as surface plasmon resonance (SPR) and bio-layer interferometry [7], kinetic isothermal titration calorimetry [8], and stopped-flow/quenched-flow [9]. Extremely successful, these techniques each have specific advantages and limitations but are overall demanding in terms of reagent quantity, in particular for the analysis of weaker interactions. More recently, single-molecule force-spectroscopy (SMFS) methods [10] have permitted high-resolution determination of association and dissociation rate constants of protein•protein [11], protein•DNA [12], and DNA·DNA interactions [13]. However, such approaches have not yet been widely adapted; indeed, protocols for attaching biomolecules to micron-size manipulators often involve significant modification of the biological system (e.g. single cysteine engineering prior to maleimide [14] or thiol conjugation [1, 2 , 15, 16], fusion of proteins through polypeptide linkers $[1,2,15])$. Here we harness DNA nanotechnology to synthesize a generic and modular scaffold compatible with rapid SMFS implementation on a wide range of interacting partners.

\section{Assembly of the dsDNA scaffold and functionalization with proteins}

The scaffold is built of three linear segments of double-strand DNA (dsDNA) that are chemically junctured together, resulting in what we name J-DNA (Fig. 1a and Supp. Fig. 1). The structure of this tool is such that two DNA ends face each other and can be pulled apart with a controlled force, as if with tweezers (dsDNA break repair could for instance be studied in this way [17]). These DNA ends, or tips, are made modular in nature and can be specifically engrafted with molecular partners for which one wishes to characterize the interaction (Fig. 1b). By monitoring the scaffold extension, for instance using magnetic tweezers to cycle between a low and a high force, one can specify whether the partners located at the tips are associated or dissociated (Fig. 2a). The leash connecting the two branches prevents the structure from disassembling in the absence of an interaction. The existence of this tether of known length also provides a mean to filter out events that do not comply with the expected gain in extension upon dissociation (Fig. $2 b$ and inset Fig. 2c) [2, 18, 19]. 
Although a variety of polymeric components have been employed to assemble such scaffolds, including polypeptide chains $[1,2,15]$ and DNA origamis $[13,18,20]$, each present specific drawbacks. Using a leash made of an unstructured sequence of a few to tens of amino acids is challenging with respect to the expression and purification of large fusion constructs [1, 2], a task that must be repeated as soon as either protein partner is to be changed. In comparison, thanks to the ease of modification and modularity of biologically-derived dsDNA, molecular partners can individually be engrafted on DNA origamis $[18,20,21]$ and on J-DNA scaffolds, making combinatorial studies more straightforward. Yet, the latter two technologies differ by their mode of assembly: whereas J-DNA tweezers are fully covalent, DNA origamis rely on the pairing of hundreds of oligonucleotides, which may result in scaffold heterogeneity or even partial disassembly upon repeated loading. Furthermore, in addition to being chemically homogeneous and hydrophilic, dsDNA is also relatively stiff, with a persistence length of $52 \mathrm{~nm}$ [22]. We thus have here a polymer of choice for gently probing molecular interactions since it allows the use of forces in the $\mathrm{fN}$ range. In comparison, leashes made of a single strand of DNA or a polypeptide (with persistence length in the Å range) can only be employed for experiments over a few $\mathrm{pN}[1,2,13,21]$. We finally note that magnetic tweezers are particularly well-suited to measurements in the low force range, and also permit simultaneous monitoring of hundreds of molecules $[23,24]$.

As a proof of principle we specifically modified the tips of the J-DNA tweezers with proteins FKBP12 and FRB whose interactions are mediated by the rapamycin class of compounds. These molecular partners constitute a good kinetic benchmarking system with both clinical and biotechnological relevance: rapamycin and its derivatives are widely-used immunosuppressive and antiproliferative drugs that modulate the $\operatorname{mTOR}$ signaling pathway $[25,26]$; they are also chemical dimerizers employed to manipulate cell function $[27,28]$. In fact, rapamycin first tightly binds to FKBP12 with an equilibrium dissociation constant $K_{D}$ on the order of $0.2-0.5 \mathrm{nM}$; the resulting binary complex then binds FRB with a $K_{D}$ reported in the $10 \mathrm{nM}$ range (Supp. Tab. 1) [29, 30, 31, 32, 33, 34].

Functionalization of the scaffold with protein partners can be carried out by a variety of methods. In a first implementation, we prepared the tips so that each of them present a 9-base 3' overhang specifically encoding for a complementary sequence that has been attached to each protein via N-terminal 4-azido-L-phenylalanine incorporation [35] followed by clicking to a 5' DBCO-modified oligonucleotide (Fig. 1b). After ligation, the J-DNA tweezers were assembled between an anti- 
digoxigenin treated glass surface and a streptavidin-coated magnetic bead thanks to the digoxigenin and biotin moieties that have been specifically incorporated at the extremity of each shanks (Fig. 1a) [36].

Force cycling in the presence of $500 \mathrm{nM}$ rapamycin shows that the scaffolded FKBP12 and FRB proteins can be repeatedly probed for association and dissociation (Fig. 2a). When the magnetic trap is switched from a low force $F^{L}$ to a high force $F^{H}$, nearly all scaffolds are found to be interacting (Fig. $2 \mathrm{~b}$ ). No association is observed in the absence of rapamycin (Supp. Fig. 2a). Durations of individual interactions derived from a single scaffold are distributed according to single-exponential statistics with a mean lifetime of $26.0 \pm 1.7 \mathrm{~s}$ (SEM, $\mathrm{n}=395$ events) for $F^{H}=1.1 \mathrm{pN}$ at $T=21.7^{\circ} \mathrm{C}$ (294.8 K) (Fig. 2c). We proceeded to collect $n=100$ to 400 rupture events from each of $N=50$ individual scaffolds, and found that the mean lifetimes of individual scaffolds are themselves distributed according to Gaussian statistics (Supp. Fig. 2b); fitting this distribution gives a central value of $\tau=28.6 \pm 3.7 \mathrm{~S}$ (SD). The dispersion in population lifetimes is interpreted as a consequence of the dispersion in the applied force, which we evaluated to be around $20 \%$ (Supp. Fig. 2c).

\section{Analysis of FKBP12 interacting with rapamycin}

At very low constant force (e.g. $F^{L}=F^{H}=0.04 \mathrm{pN}$ ) the system is seen to continuously and spontaneously associate and dissociate without a need for force cycling (Supp. Fig. 3a; mean lifetime for the closed conformation $\tau=32.4 \pm 2.7 \mathrm{~s}$ (SEM), $\mathrm{n}=182$ events analysed from $\mathrm{N}=1$ scaffold). However, when the concentration of rapamycin is decreased to $50 \mathrm{pM}$ we instead observe distinct periods of frequent interactions interspersed by very long periods where no interaction takes place (Supp. Fig. 3b). The latter idle periods reflect the absence of rapamycin from the scaffold: their duration obeys single exponential statistics (Supp. Fig. 3c) with a mean lifetime, $\tau_{i d l e}$, proportional to the inverse of rapamycin concentration (Supp. Fig. 3d). Interaction events within the active periods continue to obey singleexponential statistics (Supp. Fig. 3e), and their mean lifetime remains independent of rapamycin concentration and in the range of the value observed at $500 \mathrm{nM}$ (Supp. Fig. 3f). Finally, correcting the duration of active periods to account for impossible dissociation of a drug encased in the ternary complex, we obtain a single-exponential distribution (Supp. Fig. $3 g$ ) with a mean lifetime, $\tau_{\text {active, }}^{\text {corr }}$ also independent of rapamycin concentration (Supp. Fig. 3h).

Complementary experiments carried out using homomeric scaffolds (i.e. bearing either two FRB moieties or two FKBP12 ones), dimerized protein partners in solution, and rapamycin at less than 
250 pM identify the FKBP12-rapamycin interaction as the most stable one (Supp. Fig. 4), in agreement with the literature (Supp. Tab. 1) [29, 30, 31, 32, 33, 34]. From the numbers provided in Supp. Fig. 3 we thus determine for the FKBP12-rapamycin $\rightleftarrows$ FKBP12 + rapamycin reaction both the dissociation and association rate constants: $k_{D}=(5.3 \pm 0.57) \times 10^{-4} \mathrm{~s}^{-1}(\mathrm{SEM})$ and $k_{A}=(1.9 \pm 0.18) \times 10^{6} \mathrm{M}^{-1} \mathrm{~s}^{-1}$ (SEM) respectively. Combining both results yields $K_{D}=0.28 \pm 0.04 \mathrm{nM}(\mathrm{SEM})$. Notably, these single-molecule data are zero-force ones and agree well with reported solution or SPR measurements (Supp. Tab. 1) [29, $30,31,32,33,34]$. For instance the dissociation and association rate constants have been respectively given as $1.6 \times 10^{-3} \mathrm{~s}^{-1}$ and $5.8 \times 10^{6} \mathrm{M}^{-1} \mathrm{~s}^{-1}$ [29] or $1.4-1.6 \times 10^{-3} \mathrm{~s}^{-1}$ and $0.75-0.85 \times 10^{6} \mathrm{M}^{-1} \mathrm{~s}^{-1}$ [31].

\section{Analysis of FKBP12•rapamycin dissociating from FRB}

With this dissociation mechanism elucidated, we next completed analysis of the forcedependence of the FKBP12•rapamycin $• F R B \rightleftarrows$ FKBP12•rapamycin + FRB reaction using force cycling, at $21.7^{\circ} \mathrm{C}$ and $500 \mathrm{nM}$ rapamycin. For instance when $F^{H}$ was increased to $9.7 \mathrm{pN}$, the interaction lifetime dropped to $\tau=11.2 \pm 0.1 \mathrm{~s}$ (SD, $\mathrm{n}=18423, \mathrm{~N}=68$, Supp. Fig. $2 \mathrm{~b}$ ). In fact the dissociation rate constant of the ternary complex, $k_{D}=1 / \tau$, increases exponentially with force in agreement with the Arrhenius-Bell model: $k_{D}=A_{D} \exp \left[\left(-E_{D}+F^{H} z_{D}\right) /\left(k_{B} T\right)\right]$ (Eq. 1) with $A_{D}$ a pre-factor, $E_{D}$ the activation energy, $z_{D}$ the apparent distance to the transition state, $k_{B}$ Boltzmann's constant, and $T$ the temperature (Fig. 3a) $[37,38,39]$. By linear fitting of the data to the previous equation we obtained $z_{D}=4.4 \pm 0.1 \AA$ (SEM) and, for the dissociation rate constant at zero-force, $k_{D}^{0}=(30.8 \pm 0.4) \times 10^{-3} \mathrm{~s}^{-1}(\mathrm{SEM})-$ a value similar to the only reported one: $22 \times 10^{-3} \mathrm{~s}^{-1}$ (Supp. Tab. 1) [29]. We further note that repeated measurements roughly gave a $10 \%$ variation in $z_{D}$, but only a $3 \%$ variation in $\ln k_{D}^{0}$ (Supp. Fig. 5). Next, by performing the above measurements at different temperatures, we found that $z_{D}$ does not depend on this parameter (Fig. 3b), and we could further specify the activation energy from the dependence of $\ln k_{D}^{0}$ on $1 / T: E_{D}=58.6 \pm 1.7 \mathrm{~kJ} \mathrm{~mol}^{-1}$ (about $25 k_{B} T$ for a single molecule; Fig. $3 \mathrm{c}$ ).

To gain insight into the dissociation process, and more precisely to map the direction along which the chemical transformation is favoured [14, 16, 40, 41, 42], we generated versions of FKBP12 and FRB with internal 4-azido-L-phenylalanine labelling sites (R13AzF and E2052AzF, respectively). We then examined a total of four pulling directions by combining different pairs of protein partners labelled either at their $\mathrm{N}$-terminus or internally (Fig. 4a). For each pulling direction the intercepts of the $\ln \left[k_{D}\left(F^{H}\right)\right]$ curves with the zero-force axis differs by $\approx 10 \%$ (Fig. $4 \mathrm{~b}$ ), which is small but significantly 
superior to the $3 \%$ observed on multiplicates (Supp. Fig. 5). In parallel, the slopes are modified by nearly a factor of three (Fig. 4b). As noted in the following paragraph, mutations can result in changes in the intercepts; however, we have never observed any differences in the slopes as long as the pulling direction remained constant (Supp. Fig. 6). We thus hypothesized that these variations in $z_{D}$ reflect the fact that work supplied by the magnetic tweezers is (to first approximation) the dot product of the force vector, $\vec{F}^{H}$, with the relative displacement vector of the centres of mass of the proteins at the transition state to rupture, $\vec{Z}_{D}$ [38]. In other words, the pulling axis which is overall most parallel to the natural dissociation axis should result in the highest force sensitivity, i.e. the largest $z_{D}$ [43]. To test this model we first computed $(\vec{X}, \vec{Y})$, the best plane-of-separation between the two proteins, and we assumed $\vec{Z}_{D}$ parallel to the normal vector $\vec{Z}$ (see Methods and Fig. 4a). Then, for each pulling direction, we represented $z_{D}$ as a function of the extent to which the normalized force vector $\vec{F}_{\text {norm }}^{H}$ projects onto the $(\vec{X}, \vec{Y}, \vec{Z})$ orthonormal basis (Fig. 4c). Results indicate a strong positive correlation of $Z_{D}$ with $\vec{F}_{\text {norm }}^{H} \cdot \vec{Z}$ and a strong negative one with $\vec{F}_{\text {norm }}^{H} \cdot \vec{X}$. Consequently, the ternary complex should essentially dissociate by moving apart along the axis normal to the best plane-of-separation.

\section{Analysis of variants of the interaction and of new scaffold designs}

We first validated the ability of the method to analyse medically-relevant analogues of rapamycin: everolimus and deforolimus $[25,26] . \ln \left[k_{D}\left(F^{H}\right)\right]$ data sets were recorded in our standard conditions ( $\mathrm{N}$-terminal protein attachments, $21.7^{\circ} \mathrm{C}$, and $500 \mathrm{nM}$ of drug). For such analysis we typically collected for each force point at least $\mathrm{n}=2000$ events from at least $\mathrm{N}=10$ scaffolds. Both compounds showed a modest $30 \%$ increase in the lifetime of the ternary complex extrapolated to zero-force: $1 / k_{D}^{0}$ is equal to $32.5 \pm 0.4 \mathrm{~s}$ (SEM) for rapamycin, $41.4 \pm 0.9 \mathrm{~s}$ (SEM) for everolimus, and $44.8 \pm 1.8 \mathrm{~s}$ (SEM) for deforolimus (Supp. Fig. 6, Supp. Table 2). Secondly, we determined the effect of a number of FKBP12 and FRB mutations anticipated to modify ternary as well as binary interactions (Supp. Fig. 6, Supp. Tab. 2) $[44,45]$. The Y82F FKBP12 mutation resulted in only a slight increase (15\%) in the lifetime of the ternary complex relative to wildtype, $1 / k_{D}^{0}=37.7 \pm 1.1 \mathrm{~s}$ (SEM), while the D37V FKBP12 mutation made the ternary complex more than twice more stable relative to wildtype, $1 / k_{D}^{0}=70.6 \pm 1.3 \mathrm{~s}$ (SEM). It is interesting to note that these mutations, which have been selected from the crystal structure of the ternary complex to reduce hydrogen bonding between rapamycin and FKBP12 [44, 45], in fact result in more stable ternary complexes. We consequently investigated the association of rapamycin to FKBP12 
variants by measuring the active-period duration at low rapamycin concentration. Force-cycling experiments $\left(F^{H}=1.1 \mathrm{pN}\right.$, Supp. Fig. 7) indicate that rapamycin binding to Y82F FKBP12 is twice as longlived as to wild-type (respectively, $15600 \pm 2300$ s SEM, $n=51, N=11$ vs. $8900 \pm 1400$ s SEM, $n=41$, $\mathrm{N}=4$ ). However, rapamycin binding to D37V FKBP12 is much more unstable (134 \pm 22 s SEM, $n=38$, $\mathrm{N}=4$ ) than to wild-type. We thus identify a mutation which, intriguingly, can induce a destabilization of the binary complex in favour of a stabilization of the ternary complex. On the FRB side, the Y2105A mutation is expected to disrupt both a hydrophobic interaction with rapamycin and hydrogen bonding with FKBP12 [44, 46]. Accordingly, the lifetime of the ternary complex is dramatically reduced: $1 / k_{D}^{0}=$ $6.2 \pm 0.2 \mathrm{~s}$ (SEM). We note that a camera with a $90 \mathrm{~Hz}$ framerate allows us to observe short-lived $(\approx 50$ ms) interactions between Y2105A FRB and the FKB12-rapamycin complex (Supp. Fig. 8).

Finally, we introduce two modifications of the scaffold that illustrate the modularity of our approach. First, in order to make SMFS experiments universally accessible to other biological systems, we devised a second engrafting strategy based on standard protein expression tools: we fused the genetically-encoded CLIP- and SNAP-tags to the N-termini of the proteins of interest (Fig. 5a) [47, 48]. These protein tags bind specifically and covalently to the benzylcytosine (BC) and benzylguanine (BG) small molecules, respectively. Thus, by preparing J-DNA tweezers bearing a BC group at one DNA tip and a BG group at the other, we could simply assemble CLIP-FKBP12 and SNAP-FRB onto the scaffold by incubation. Results obtained with this approach are identical to those obtained with the N-terminal 4azido-L-phenylalanine labelling strategy (Fig. 5b), proving that SMFS outcomes are robust with respect to tip modifications. We further note that the CLIP- and SNAP-protein tags are already widely accepted in cellular biology, where they are used for fluorescent labelling and monitoring of proteins in vivo [49]; therefore, we anticipate both rapid adoption as well as fast implementation of this alternative engraftment strategy.

In a second implementation we modified the leash length of the original scaffold so as to be able to tune association efficacy. During cycling experiments the likelihood of detecting an interaction decreased from 85 to $6 \%$ when we increased the leash length from $\approx 0.7$ to $\approx 2.7 \mathrm{kbp}$ (Supp. Fig. 9), as expected for a dilution effect. In fact, J-factor measurements indicate that the 700 base-pair value of our initial design is certainly an optimal one with respect to complex formation at nearly zero-force: smaller loops are associated with a high enthalpic cost in term of bending energy whereas larger loops are associated with a high entropic cost because distant molecular partners have to enter in collision [50]. 


\section{Conclusions}

The single-molecule tool we have developed provides precise, accurate, and model-independent measurements of the lifetime of rapamycin protein interactions. Rate constants are determined with error bars lower than $10 \%$ and the obtained values are in agreement with those found in the literature (Supp. Tab. 1, Supp. Tab. 2) [29, 31]. Importantly, our experiments necessitate less than $1 \%$ of the amount of protein reagents required for any other kinetic method: 250 samples can be prepared with as little as $12 \mathrm{fmol}$ of SNAP- and CLIP-tag conjugates, which is largely enough to measure the three rate constants we have determined for the binary and ternary complexes (see Methods). At present, $50 \mathrm{~ms}$ to $5000 \mathrm{~s}$ long rupture events can be assessed with an applied force between $30 \mathrm{fN}$ to $10 \mathrm{pN}$, covering a wide range of biological interactions. Importantly, the dsDNA leash uniquely enables one to probe engrafted complexes using extremely low forces, thereby advantageously placing oneself in solution-like conditions. All other performances are setup-dependent and can readily be improved (magnetic actuation down to $20 \mathrm{~ms}$, force up to $100 \mathrm{pN}$ ). In fact, J-DNA scaffolds are also expected to comply with SMFS apparatuses having different working ranges (e.g. optical tweezers, atomic force microscope).

The modularity and simplicity of J-DNA tweezers make established single-molecule metrics (i.e. position of the transition state in space and energy, direction of the dissociation axis) accessible to researchers in drug design. Direct application can readily be foreseen for a large variety of active compounds stabilizing protein protein interactions through the formation of a ternary complex (drugs, among which immunosuppressants $[25,51]$ and PROTACs $[52,53]$, as well as dimerizers used in chemical biology $[27,28])$. In addition, the ability to scrutinize the force-dependence of dissociation kinetics should be significant for medicines targeting the cytoskeleton [51] or mediating recognition between cancer and immune cells [54]. Ultimately, other classes of nanomaterials, both organic and inorganic, should be amenable to such investigations. 


\section{References}

[1] L. Rognoni, J. Stigler, B. Pelz, J. Ylanne, and M. Rief. Dynamic force sensing of filamin revealed in single-molecule experiments. Proc. Natl. Acad. Sci. (USA), 109:19679-19684, 2012.

[2] J. Kim, C.-Z. Zhang, X. Zhang, and T.A. Springer. A mechanically stabilized receptor-ligand flexbond important in the vasculature. Nature, 466:992-995, 2010.

[3] B.T. Marshall, M. Long, J.W. Piper, T. Yago, R.P. McEver, and C. Zhu. Direct observation of catch bonds involving cell-adhesion molecules. Nature, 423:190-193, 2003.

[4] R.A. Copeland. The drug-target residence time model: A 10-year perspective. Nat. Rev. Drug Disc., 15:87-95, 2016.

[5] D. A. Schuetz, W. E. A. de Witte, Y. C. Wong, B. Knasmueller, L. Richter, D. B. Kokh, S. K. Sadiq, R. Bosma, I. Nederpelt, L. H. Heitman, E. Segala, M. Amaral, D. Guo, D. Andres, V. Georgi, L. A. Stoddart, S. Hill, R. M. Cooke, C. De Graaf, R. Leurs, M. Frech, R. C. Wade, E. C. M. de Lange, A. P. Ijzerman, A. Muller-Fahrnow, and G. F. Ecker. Kinetics for drug discovery: An industry-driven effort to target drug residence time. Drug Disc. Today, 22:896-911, 2017.

[6] D.C. Swinney. Applications of binding kinetics to drug discovery. Curr. Op. Pharm. Med., 22:2334, 2008.

[7] Y. Fang. Ligand-receptor interaction platforms and their applications for drug discovery. Expert Opin. Drug. Discov., 7:969-988, 2012.

[8] D. Burnouf, E. Ennifar, S. Guedich, B. Puffer, G. Hoffmann, G. Bec, F. Disdier, M. Baltzinger, and P. Dumas. kinITC: A new method for obtaining joint thermodynamic and kinetic data by isothermal titration calorimetry. J. Am. Chem. Soc., 134:559-565, 2012.

[9] J.F. Eccleston, S.R. Martin, and M.J. Schilstra. Rapid kinetic techniques. Meth. Cell. Biol., 84:445477, 2008.

[10] S. Garcia-Manyes and A.E.M. Beedle. Steering chemical reactions with force-dependent. Nat. Rev. Chem. 1:\#83, 2017.

[11] J.R. Sellers and C. Veigel. Direct observation of the myosin-Va power stroke and its reversal. Nat. Struct. Mol. Biol., 17:590-595, 2010.

[12] M.A. Hall, A. Shundrovsky, L. Bai, R.M. Fulbright, J.T. Lis, and M.D. Wang. High-resolution dynamic mapping of histone-DNA interactions in a nucleosome. Nat. Struct. Mol. Biol., 16:124-129, 2009.

[13] F. Kilcherr, C. Wachauf, B. Pelz, M. Rief, M. Zacharias, and H. Dietz. Single-molecule dissection of stacking forces in DNA. Science, 353:aaf5508 1-9, 2016.

[14] C. Schoeler, R.C. Bernardi, K.H. Malinowska, E. Durner, W. Ott, E.A. Bayer, K. Schulten, M.A. Nash, and H.E. Gaub. Mapping mechanical force propagation through biomolecular complexes. Nano Lett., 15:7370-7376, 2015.

[15] Y. Gao, S. Zorman, G. Gundersen, Z. Xi, L. Ma, G. Sirinakis, J.E. Rothman, and Y. Zhang. Single reconstituted neuronal SNARE complexes zipper in three distinct stages. Science, 337:1340-1343, 2012. 
[16] H. Dietz, F. Berkemeier, M. Bertz, and M. Rief. Anisotropic deformation response of single protein molecules. Proc. Natl. Acad. Sci. U.S.A., 103:12724-12728, 2006.

[17] J.L. Wang, C. Duboc, Q. Wu, T. Ochi, S. Liang, S.E. Tsutakawa, S.P. Lees-Miller, M. Nadal, J.A. Tainer, T.L. Blundell, and T. R. Strick. Dissection of DNA double-strand-break repair using novel singlemolecule forceps. Nat. Struct. Mol. Biol., 25:482-487, 2018.

[18] K. Halvorsen, D. Schaak, and W. P. Wong. Nanoengineering a single-molecule mechanical switch using DNA self-assembly. Nanotechnology, 22:494005, 2011.

[19] K. C. Johnson and W. E. Thomas. How do we know when single-molecule force spectroscopy really tests single bonds? Biophys. J., 114:2032-2039, 2018.

[20] D. Koirala, P. Shrestha, T. Emura, K. Hidaka, S. Mandal, M. Endo, H. Sugiyama, and Mao. H. Single-molecule mechanochemical sensing using DNA origami nanostructues. Angew. Chem. Int. Ed., 53:8137-8141, 2014.

[21] P. C. Nickels, B. Wunsch, P. Holzmeister, W. Bae, L. M. Kneer, D. Grohmann, P. Tinnefeld, and T. Liedl. Molecular force spectroscopy with a DNA origami-based nanoscopic force clamp. Science, 354:305-307, 2016.

[22] C. Bouchiat, M.D. Wang, S.M. Block, J.-F. Allemand, T.R. Strick, and V.Croquette. Estimating the persistence length of a worm-like chain molecule from force-extension measurements. Biophys. J., 76:409-413, 1999.

[23] N. Ribeck and O.A. Saleh. Multiplexed single-molecule measurements with magnetic tweezers. Rev. Sci. Instrum., 79:094301, 2008.

[24] I. De Vlaminck, T. Henighan, M.T.J. van Loenhout, I. Pfeiffer, J. Huijts, J.W.J Kerssemakers, A.J. Katan, A. van Langen-Suurling, E. van der Drift, C. Wyman, and C. Dekker. Highly parallel magnetic tweezers by targeted DNA tethering. Nano Lett., 11:5489-5493, 2011.

[25] F. Liu, Y.-Q. Wang, L. Meng, M. Gu, and R.-Y. Tan. FK506-binding protein 12 ligands: a patent review. Expert Opin. Ther. Patents, 23:1435-1449, 2013.

[26] D. Benjamin, M. Colombi, C. Moroni, and M. N. Hall. Rapamycin passes the torch: a new generation of mTOR inhibitors. Nat. Rev. Drug Discovery, 10:868-880, 2011.

[27] M. Putyrski and C. Schultz. Protein translocation as a tool: The current rapamycin story. FEBS Lett., 586:2097-2105, 2012.

[28] S. Voss, L. Klewer, and Y.-W. Wu. Chemically induced dimerization: reversible and spatiotemporal control of protein function in cells. Curr. Opin. Chem. Biol., 28:194-201, 2015.

[29] L.A. Banaszynski, C.W. Liu, and T.J. Wandless. Characterization of the FKBP.rapamycin.FRB ternary complex. J. Am. Chem. Soc., 127:4715-4721, 2005.

[30] B. E. Bierer, P. S. Mattila, R. F. Standaert, L. A. Herzenberg, S. J. Burakoff, G. Crabtree, and S. L. Schreiber. Two distinct signal transmission pathways in lymphocytes-T are inhibited by complexes formed between an immunophilin and either FK506 or rapamycin. Proc. Natl. Acad. Sci. (USA), 87:92319235, 1990. 
[31] M.A. Wear and M.D. Walkinshaw. Determination of the rate constants for the FK506 binding protein/rapamycin interaction using surface plasmon resonance: an alternative sensor surface for $\mathrm{Ni2+-}$ nitrilotriacetic acid immobilization of His-tagged proteins. Anal. Biochem., 371:250-252, 2007.

[32] C. Kozany, A. Marz, C. Kress, and F. Hausch. Fluorescent probes to characterise FK506-binding proteins. Chembiochem, 10:1402-1410, 2009.

[33] T. Tamura, Y. Kioi, T. Miki, S. Tsukiji, and I. Hamachi. Fluorophore labeling of native FKBP12 by ligand-directed tosyl chemistry allows detection of its molecular interactions in vitro and in living cells. J. Am. Chem. Soc., 135:6782-6785, 2013.

[34] C. Lu and Z. X. Wang. Quantitative analysis of ligand induced heterodimerization of two distinct receptors. Anal. Chem., 89:6926-6930, 2017.

[35] J.W. Chin, S.W. Santoro, A.B. Martin, D.S. King, L. Wang, and P.G. Schultz. Addition of p-azido-Lphenylalanine to the genetic code of Escherichia coli. J. Am. Chem. Soc., 124:9026-9027, 2002.

[36] C. Duboc, J. Fan, E.T. Graves, and T.R. Strick. Preparation of DNA substrates and functionalized glass surfaces for correlative nanomanipulation and colocalization (NanoCOSM) of single molecules. Meth. Enz., 582:275-296, 2017.

[37] M. Schlierf, H. Li, and J.M. Fernandez. The unfolding kinetics of ubiquitin captured with singlemolecule force-clamp techniques. Proc. Natl. Acad. Sci. (USA), 101:7299-7304, 2004.

[38] E. Evans. Probing the relation between force - lifetime - and chemistry in single molecular bonds. Annu. Rev. Biophys. Biomol. Struct., 30:105-128, 2001.

[39] I Popa, J.M. Fernandez, and S. Garcia-Manyes. Direct quantification of the attempt frequency determining the mechanical unfolding of ubiquitin protein. J. Biol. Chem., 286:31072-31079, 2011.

[40] D.J. Brockwell, E. Paci, R.C. Zinober, G.S. Beddard, P.D. Olmsted, D.A. Smith, R.N. Perham, and S.E. Radford. Pulling geometry defines the mechanical resistance of a beta-sheet protein. Nat. Struct. Biol., 10:731-737, 2003.

[41] M. Carrion-Vazquez, H. Li, H. Lu, P.E. Marszalek, A.F. Oberhauser, and J.M. Fernandez. The mechanical stability of ubiquitin is linkage dependent. Nat. Struct. Biol., 10:738-743, 2003.

[42] B. Jagannathan, P.J. Elms, C. Bustamante, and S. Marqusee. Direct observation of a forceinduced switch in the anisotropic mechanical unfolding pathway of a protein. Proc. Natl. Acad. Sci. (USA), 109:17820-17825, 2012.

[43] S.R.K. Ainavarapu, A.P. Wiita, L. Dougan, E. Uggerud, and J.M. Fernandez. Single-molecule force spectroscopy measurements of bond elongation during a bimolecular reaction. J. Am. Chem. Soc., 130:6479-6487, 2008.

[44] J. W. Choi, J. Chen, S. L. Schreiber, and J. Clardy. Structure of the FKBP12-rapamycin complex interacting with the binding domain of human FRAP. Science, 273:239-242, 1996.

[45] M. T. DeCenzo, S. T. Park, B. P. Jarrett, R. A. Aldape, O. Futer, M. A. Murcko, and D. J. Livingston. FK506-binding protein mutational analysis: Defining the active-site residue contributions to catalysis and the stability of ligand complexes. Protein Eng., 9:173-180, 1996. 
[46] M. Leone, K. J. Crowell, J. H. Chen, D. W. Jung, G. G. Chiang, S. Sareth, R. T. Abraham, and M. Pellecchia. The FRB domain of mTOR: NMR solution structure and inhibitor design. Biochemistry, 45:10294-10302, 2006.

[47] A. Keppler, M. Kindermann, S. Gendreizig, H. Pick, H. Vogel, and K. Johnsson. Labeling of fusion proteins of 06-alkylguanine-DNA alkyltransferase with small molecules in vivo and in vitro. Methods, 32:437-444, 2004.

[48] M.J. Hinner and K. Johnsson. How to obtain labeled proteins and what to do with them. Curr. Opin. Biotechnol., 21:766-776, 2010.

[49] A. Gautier, A. Juillerat, C. Heinis, I.R. CorrÃăa, M. Kindermann, F. Beaufils, and K. Johnsson. An engineered protein tag for multiprotein labeling in living cells. Chem. Biol., 15:128-136, 2008.

[50] J-F. Allemand, S. Cocco, N. Douarche, and G. Lia. Loops in DNA: an overview of experimental and theoretical approaches. Eur. Phys. J. E Soft Matter, 19:293-302, 2006.

[51] D. Bier, P. Thiel, J. Briels, and C. Ottmann. Stabilization of protein-protein interactions in chemical biology and drug discovery. Prog. Biophys. Mol. Biol., 119:10-19, 2015.

[52] T. K. Neklesa, J. D. Winkler, and C. M. Crews. Targeted protein degradation by protacs. Pharmacol. Ther., 174:138-144, 2017.

[53] M. J. Roy, S. Winkler, S. J. Hughes, C. Whitworth, M. Galant, W. Farnaby, K. Rumpel, and A. Ciulli. SPR-measured dissociation kinetics of PROTAC ternary complexes influence target degradation rate. ACS Chem. Biol., 14:361-368, 2019.

[54] J. Del Bano, P. Chames, D. Baty, and B. Kerfelec. Taking up cancer immunotherapy challenges: Bispecific antibodies, the path forward? Antibodies, 5:23, 2016. 

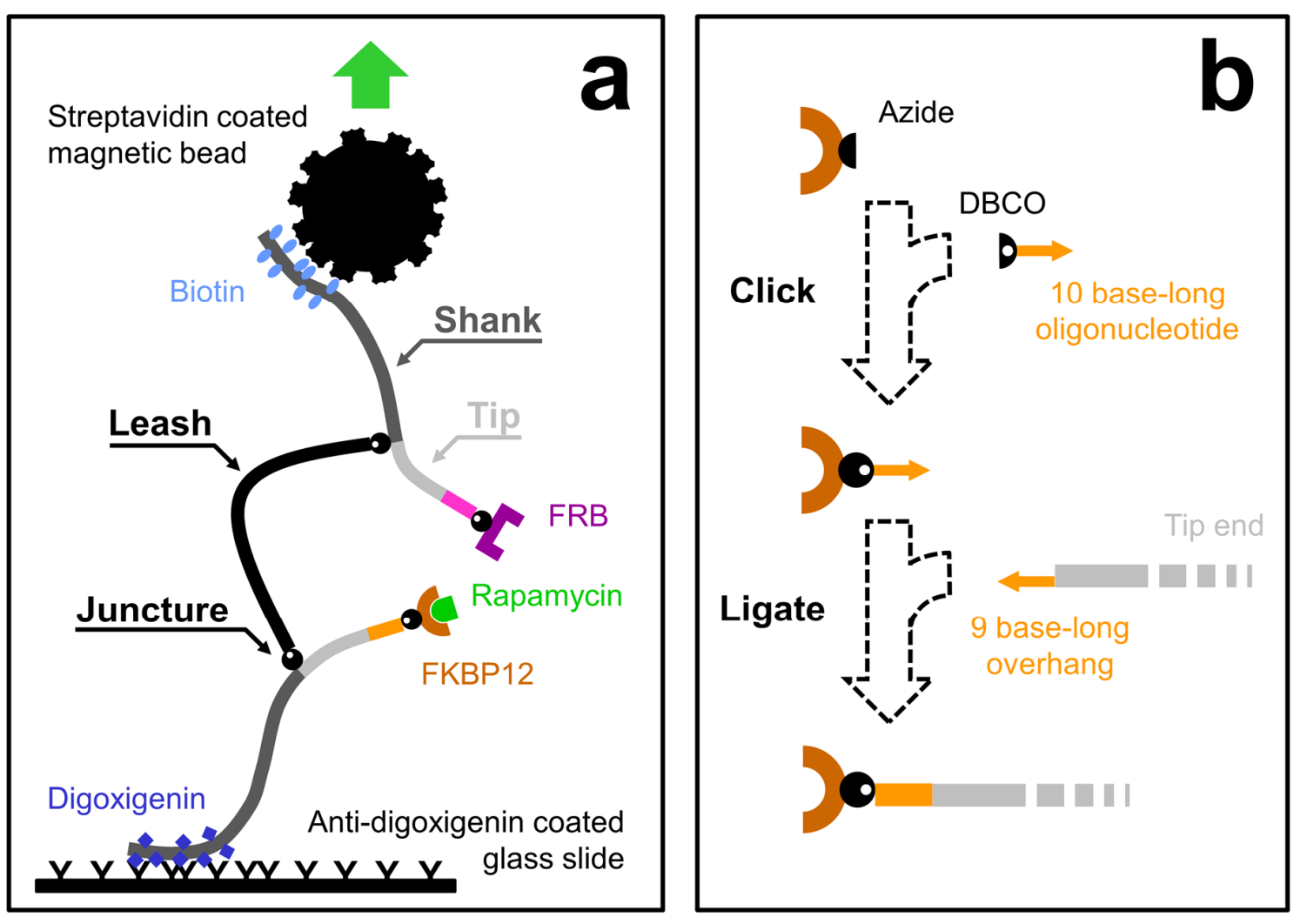

Fig. 1 | Structure of the junctured-DNA (J-DNA) tweezers and first generic strategy used to attach a given protein at a given tip. a, The molecular scaffold is constituted of three linear dsDNA segments: two branches, divided into shank and tip, held together by a leash. Freely-swivelling junctures between branches and leash minimize transmission of steric constraints between the segments. One of the shanks is attached to a glass surface and the other one to a magnetic bead, the two handles of the micron-size manipulator we are using, i.e. magnetic tweezers. Each tip can be specifically functionalized with a given biomolecule, here either the FRB domain of the mTOR kinase or the FKBP12 immunophilin. In our first implementation the length of the dsDNA segments is around 700 and 1300 bp for the shanks, 41 and $47 \mathrm{bp}$ for the tip, and $690 \mathrm{bp}$ for the leash (drawing not to scale). b, For engraftment an azide moiety is first introduced at a desired location in the peptidic backbone using unnatural amino acid incorporation. This chemical group is next reacted with an oligonucleotide bearing a 5' DBCO group. The single-strand DNA sequence now connected to the protein is complementary to the one present at one of the tip of the tweezers. The protein can thus be specifically addressed by hybridization before undergoing covalent enzymatic ligation. 


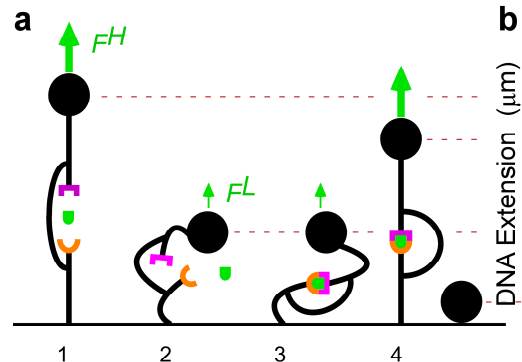

b

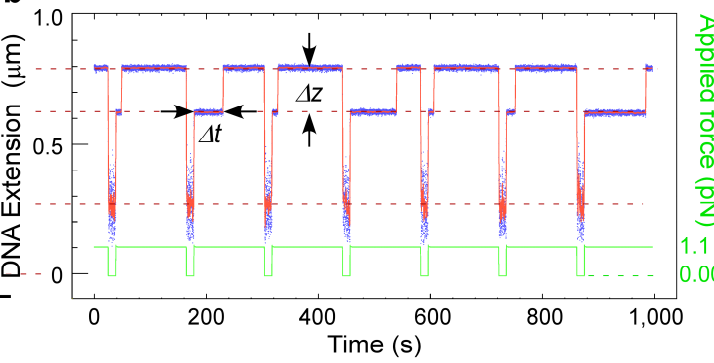

C

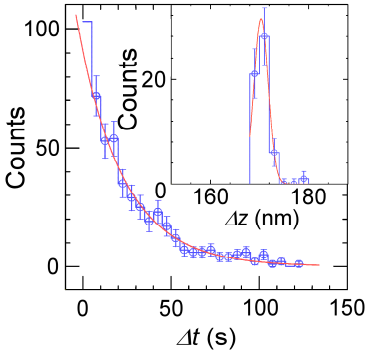

Fig. 2 | Experiment design and initial results on a single molecule. a, Sketch of the force-cycling procedure applied to the J-DNA scaffold using magnetic tweezers. The scaffold is engrafted with FKBP12 and FRB, and rapamycin is present in solution at $500 \mathrm{nM}$, a saturating concentration for the FKBP12 - rapamycin interaction $\left(K_{D}=0.2-0.5 \mathrm{nM}\right)$ but not for the rapamycin $\cdot$ FRB one $\left(K_{D}>1 \mu \mathrm{M}\right)$ (Supp. Tab. 1) [29, 30, 31, 32, 33, 34]. A reference bead stuck on the surface is monitored to correct for microscope drift. (1) The tweezers are initialized in a high-force, high-extension state. (2) The force is lowered, bringing the tips into proximity and (3) allowing FKBP12 and FRB to bind via their interaction with rapamycin. (4) The force is returned to its initial value and the resulting DNA extension reports on the association state of the proteins. Eventually, the scaffold recovers its initial high-extension state (1) after dissociation of the ternary complex. $\mathbf{b}$, Representative time-trace of DNA extension as the tweezers are cycled between a low force, $F^{L}=0.001 \mathrm{pN}$, for $15 \mathrm{~s}$ and a high force, $F^{H}=1.1 \mathrm{pN}$, for $125 \mathrm{~s}$. Each interaction event is characterized by its duration, $\Delta t$, and by the change in DNA scaffold extension following tweezers opening, $\Delta z$. Data were collected at $T=21.7^{\circ} \mathrm{C}$ in the mTOR buffer ( $20 \mathrm{mM} \mathrm{K} \cdot \mathrm{HEPES}$, $\mathrm{pH}$ 7.8, $100 \mathrm{mM} \mathrm{KCl}, 5 \mathrm{mM} \mathrm{MgCl}$, $0.1 \%$ Tween-20, $0.5 \mathrm{mg} / \mathrm{mL} \mathrm{BSA}, 2 \mathrm{mM}$ DTT). Blue points: raw data obtained at $31 \mathrm{~Hz}$. Red line: raw data averaged over $1 \mathrm{~s}$. Green line: force modulation pattern. c, The histogram of interaction durations is fit by a single-exponential distribution, yielding $26.0 \pm 1.7 \mathrm{~s}$ for the mean lifetime of this scaffold in the closed configuration (SEM, $n=395$ rupture events analysed). Extension changes (inset) are fit to a Gaussian statistic, providing a mean value of $170 \pm 1.5 \mathrm{~nm}$ (SD, $\mathrm{n}=$ 56 rupture events analysed). Red lines are fits to the data. 

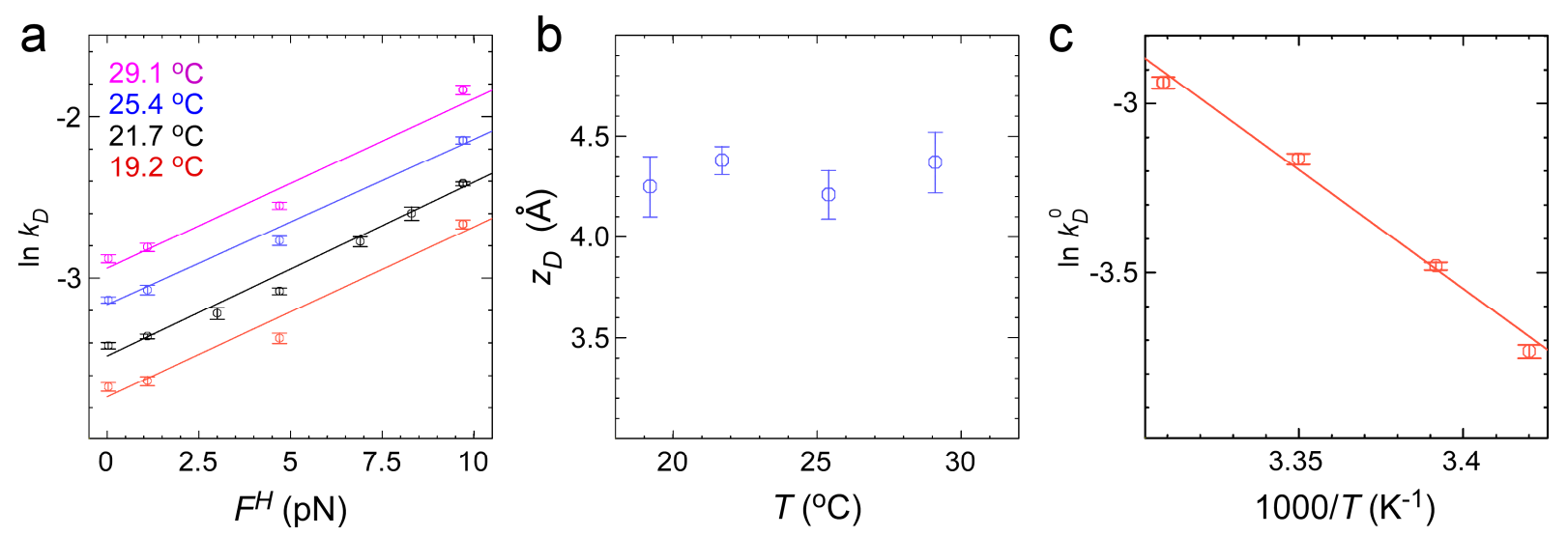

Fig. 3 |Influence of force and temperature on dissociation of the FKBP12-rapamycin-FRB complex. a, Force-dependence, at four different temperatures, of the dissociation rate constant. All $k_{D}$ were measured upon force cycling except the ones at $0.03 \mathrm{pN}$, which were determined upon analysis of spontaneous fluctuations. Linear fitting according to the Arrhenius-Bell model provides, for each dataset, $z_{D} / k_{B} T$ as slope and $\ln k_{D}^{0}=\ln A_{D}-E_{D} / k_{B} T$ (Eq. 2) as intercept with the $F^{H}=0$ axis. b, Temperature-dependence of the apparent position of the transition state. $\mathbf{c}$, Temperature-dependence of the zero-force dissociation rate constant. Linear fitting yields for the activation energy $E_{D}=58.6 \pm 1.7$ $\mathrm{kJ} \mathrm{mol}^{-1}$ (SEM). Data were collected in the mTOR buffer supplemented with $500 \mathrm{nM}$ rapamycin; error bars represent SEM. 

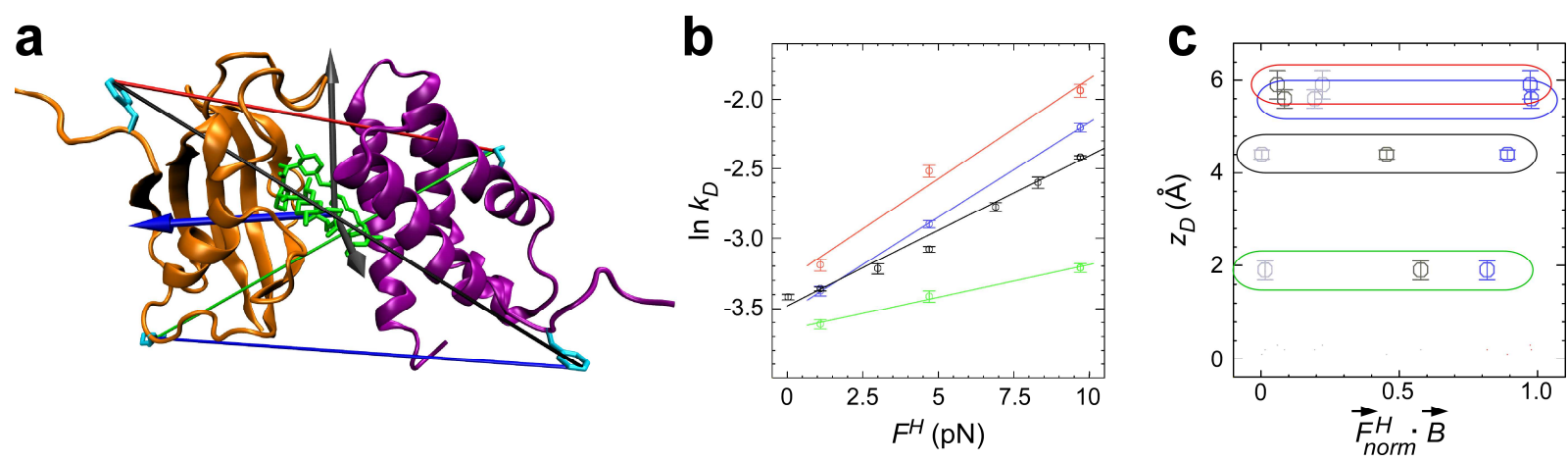

Fig. 4 | Influence of the pulling direction on dissociation of the FKBP12-rapamycin-FRB complex. a, Structure of the ternary complex with the positions of the mutated amino acids indicated in cyan and the directions of the force vectors materialized by green, black, blue, and red lines. Arrows represent a set of basis vectors oriented so as to emphasize the best plane-of-separation between the two proteins ( $\vec{X}$ and $\vec{Y}$ are grey and in-plane, $\vec{Z}$ is blue and normal). b, Force-dependence of the dissociation rate constant. Curves are color-coded as per the structural depiction in (a). The black dataset is that for the original labelling position presented in Fig. 3a. Linear fitting according to the Arrhenius-Bell model yields: $z_{D}=1.9 \pm 0.2 \AA$ and $\ln k_{D}^{0}=-3.65 \pm 0.03$ (SEM) for the green curve; $z_{D}=4.4 \pm 0.1 \AA$ and $\ln k_{D}^{0}=-3.48 \pm$ 0.01 (SEM) for the black curve; $z_{D}=5.6 \pm 0.2 \AA$ and $\ln k_{D}^{0}=-3.53 \pm 0.03$ (SEM) for the blue curve; and $z_{D}=5.9 \pm 0.3 \AA$ and $\ln k_{D}^{0}=-3.30 \pm 0.04$ (SEM) for the red curve. c, Plot of the apparent distance to the transition state as a function of the projection of the normalized force vector $\vec{F}_{n o r m}^{H}=\vec{F}^{H} /\left\|\vec{F}^{H}\right\|$ on the different unit vectors of the $(\vec{X}, \vec{Y}, \vec{Z})$ basis. Each coloured pill corresponds to a given pulling direction, and includes three points. For each pill, the abscissae are $\vec{F}_{\text {norm }}^{H} \cdot \vec{B}$ with either $\vec{B}=\vec{X}$ (dark grey), $\vec{Y}$ (light grey), or $\vec{Z}$ (blue). Data were collected at $21.7^{\circ} \mathrm{C}$ in the mTOR buffer supplemented with $500 \mathrm{nM}$ rapamycin; error bars represent SEM; all $k_{D}$ were measured upon force cycling $\left(F^{L}=0.001 \mathrm{pN}\right)$ except the one at $F^{H}=F^{L}=0.03 \mathrm{pN}$, which was determined upon analysis of spontaneous fluctuations. 

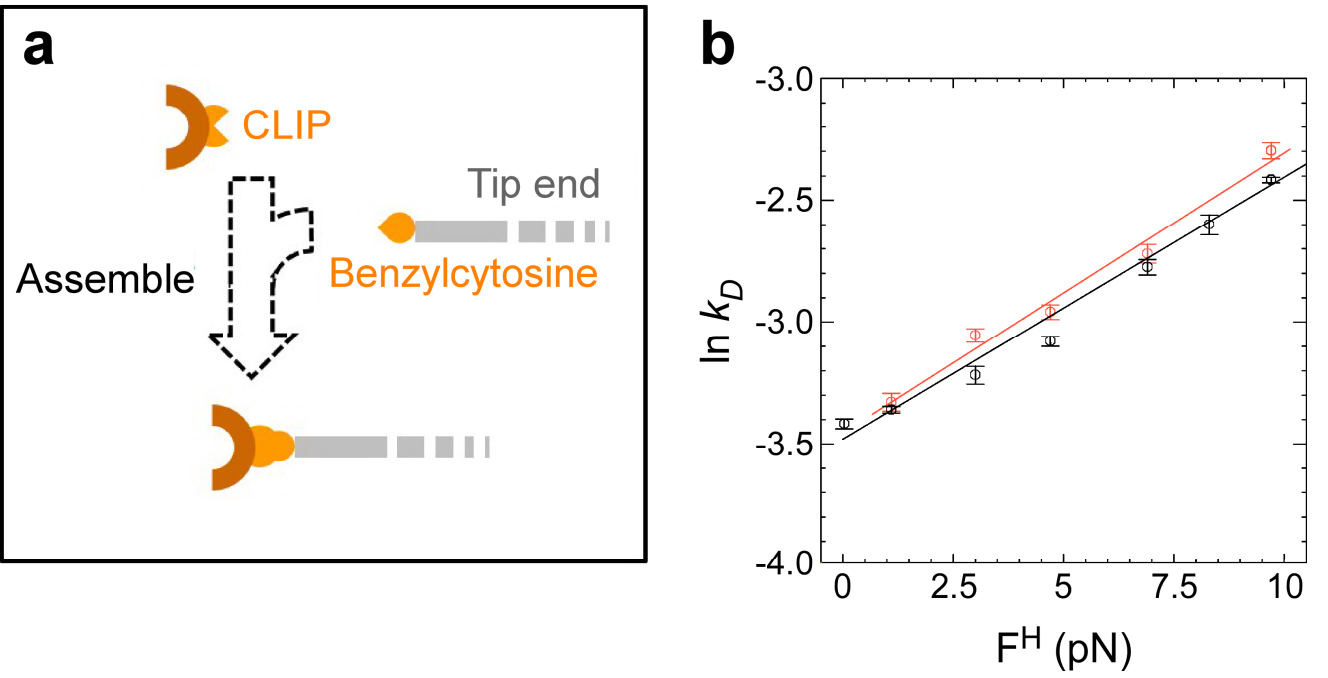

Fig. 5 | Second generic strategy used to engraft a given protein at a given tip of the J-DNA tweezers. a, The protein of interest is fused to a genetically-encoded protein tag complementary to a small molecule located at one of the tip of the scaffold. The two entities react together to form a covalent adduct. The present illustration is given for the CLIP/benzylcytosine couple but the SNAP/benzylguanine one works similarly. $\mathbf{b}$, Force-dependence of the dissociation rate constant of the ternary FKBP12 -rapamycin $\cdot F R B$ complex engrafted via CLIP- and SNAP-tags: $z_{D}=4.7 \pm 0.2 \AA$ and $\ln k_{D}^{0}=-3.45 \pm 0.03$ (SEM) for the red curve. These measurements are compared with the ones obtained for protein components engrafted via oligonucleotidic intermediates (same dataset as the one presented in Fig. 3a: $z_{D}=4.4 \pm 0.1 \AA$ and $\ln k_{D}^{0}$ $=-3.48 \pm 0.01$ (SEM) for the black curve). Data were collected at $21.7{ }^{\circ} \mathrm{C}$ in the mTOR buffer supplemented with $500 \mathrm{nM}$ rapamycin; error bars represent SEM; all $k_{D}$ were measured upon force cycling $\left(F^{L}=0.001 \mathrm{pN}\right)$ except the one at $F^{H}=F^{L}=0.03 \mathrm{pN}$, which was determined upon analysis of spontaneous fluctuations. 


\section{Acknowledgements}

This work was supported by the following grants: T-DropTwo (Labex NanoSaclay); T-DropThree (Idex Paris-Saclay); NanoRep (PSL University); Gephyrip (Labex Memolife); J-DNA (PSL-Valorisation). T.R.S. is part of the "Equipe Labellisée" program of the Ligue Nationale Contre la Cancer. H.K.W.S. and J.L.W. respectively acknowledge the National Science Foundation and the China Scholarship Council for PhD fellowships. We are finally thankful to Alix Thomas and Leah Friedman for preliminary experiments, as well as to the groups of Jie Yan and Felix Hausch for discussions on unpublished data.

\section{Author Contributions}

D.K., T.R.S., and C.G. conceived the experiments; J.L.W. contributed unique reagents; D.K. and M.F. prepared reagents and carried out measurements; D.K. and T.R.S. carried out primary data analysis; D.K., H.K.W.S., V.S.P., T.R.S., and C.G. carried out advanced data analysis and modelling; D.K., T.R.S., and C.G. wrote the paper.

\section{Competing interests}

The authors declare no financial or non-financial competing interests.

\section{Additional Information}

Supplementary information is available in the online version of the paper. Reprints and permission information is available online at www.nature.com/reprints. Correspondence and requests for materials should be addressed to either T. R. Strick or C. Gosse. 


\section{Methods}

\section{Synthesis of J-DNA tweezers}

\section{General strategy}

J-DNA scaffolds were prepared as described previously [17] with the exception of a few modifications (see Supp. Fig. 1 for a graphical depiction). Briefly, the tweezers still consist of two linear dsDNA segments, the two branches, connected by a third one, the leash. However, those three respective elements are now $\approx 690,1330$, and $690 \mathrm{bp}$ long (a variant with a leash $\approx 2.7 \mathrm{kbp}$ in size has also been used but only for a single experiment, the one of Supp. Fig. 9). In addition to those new dimensions, the tips of the branches are now functionalized and serve as attachment points for the molecular partners: in a first implementation two single-strand overhangs generated by $\mathrm{Nb} . \mathrm{BbvCl}$ nicking can be used for ligation of oligonucleotide-conjugated proteins; in a second implementation incorporation of benzylguanine (BG) and benzylcytosine (BC) allow for direct immobilization of SNAPand CLIP-tagged proteins $[49,55]$.

\section{Oligonucleotides sequences (5' to 3' direction)}

$\mathrm{L}_{1}$

xCCATGGGCATACTGATCGGTAGGG

$\mathrm{L}_{2}$

XGAGCCAAGACGCCTCCATCCATGCA

$\mathrm{TS}_{1}$

ATATGAGGCTGAGGAAGCGGTTAGCTCCTTCGGTCCTCCGATCGTTGYCAGAAGTAAGTTGGCCG CAG

$\mathrm{TS}_{2}$

TGTAAGAGCTGAGGCGGTGACCAATATCTACAACATCAGCCYTGGTATCCAGCGTGATG

O1-Comp

Pho-CGCGCCCTACCGATCAGTATGCCCATGGA 
O2-Comp

Pho-TGGATGGAGGCGTCTTGGCTCA

Charo-3600-MluI

GAGAGAACGCGTTACCTGTCCGCCTTTCTCCCTTCGGG

Charo-4230-SbfI

GAGAGACCTGCAGGCCTCACTGATTAAGCATTGGTAACTGTCAGACC

$\mathrm{TS}_{3}$

zGTAAGAGCTGAGGCGGTGACCAATATCTACAACATCAGCCYTGGTATCCAGCGTGATG

$\mathrm{TS}_{4}$

QTCTCAAGCTGAGGAAGCGGTTAGCTCCTTCGGTCCTCCGATCGTTGYCAGAAGTAAGTTGGCCG CAG

In the present notation $\mathbf{X}$ indicates DBCO-dT (Glen Research), Y Azido-dT, Pho a 5' phosphate group, $\mathbf{z} \mathrm{O}^{2}$-benzylcytosine-dT (New England Biolabs/Trilink), and $\mathbf{Q} \mathrm{O}^{6}$-benzylguanine-dA (New England Biolabs/Trilink). Furthermore, the underlined sequence is the $\mathrm{Nb} . \mathrm{BbvCl}$ nicking site.

J-DNA tweezers with a 690 bp leash and functionalized by Nb.BbvCl-generated ssDNA overhangs

Oligonucleotides $L_{1}, L_{2}, T S_{1}$, and $T_{S}$ were synthesized by Trilink Inc. and resuspended in formamide (Merck) at $37^{\circ} \mathrm{C}$, so as to obtain a $10 \mu \mathrm{g} / \mu \mathrm{L}$ final concentration. Typically, $\approx 100 \mu \mathrm{g}$ of each $\mathrm{L}_{i}$ was combined with an equimolar amount of the associated $\mathrm{TS}_{\mathrm{i}}$ and incubated $12 \mathrm{~h}$ at $25^{\circ} \mathrm{C}$, in order to yield the corresponding $J_{i}$ product by click-chemistry coupling (Step a1 in Supp. Fig. 1). Both $J_{1}$ and $\mathrm{J}_{2}$ branched oligonucleotides were then purified by electrophoresis on an $8 \mathrm{M}$ urea, $8 \%$ acrylamide/bisacrylamide (29:1) gel as previously described [17]. Subsequently, $J_{1}$ and $J_{2}$ were used as primers in the PCR amplification of a $\approx 2.0 \mathrm{kbp}$ fragment of the Charomid 9-5 $\Delta \mathrm{Sbfl}$ template with the Expand High Fidelity polymerase system (Roche) (Step a2 in Supp. Fig. 1) - the used template is a derivative of the Charomid 9-5 plasmid in which the native Sbfl site has been removed by Sbfl digestion, fill-in, and religation with the Quick Blunting kit (New England Biolabs). The resulting branched PCR product, which is the precursor of the tips and shanks of the J-DNA tweezers, was purified using the NucleoSpin Gel and PCR Clean-up kit (Macherey-Nagel). 
In parallel, the leash precursor was synthesized as follow. Its $\approx 630$ bp backbone was amplified by PCR using the Platinum Pfx DNA polymerase (Invitrogen), the Charomid 9-5 $\Delta$ Sbfl template, and the Charo-3600-MluI and Charo-4230-SbfI primers. The blunt-end PCR product was then digested with Sbfl and cloned into the pUC18 vector using the Sbfl/Smal restriction site. The resulting DNA plasmid, 690_pUC18, was amplified and purified using the NucleoBond Xtra maxiprep kit (MachereyNagel), and digested with the Mlul and Sbfl restriction enzymes. The digestion products were separated using by electrophoresis on a $1 \%$ agarose gel and extracted using the NucleoSpin Gel and PCR Clean-up kit (Macherey-Nagel).

Next, the $L_{1}$ and $L_{2}$ oligonucleotides of the branched PCR product, which have not participated in the amplification reaction, were annealed in equimolar ratio to the $01-$ Comp and $02-$ Comp oligonucleotides, respectively (Step a3 in Supp. Fig. 1). This reaction took place at a final concentration of $\approx 270 \mathrm{nM}$ in each strand, for $1 \mathrm{~h}$ at room temperature in $1 \times$ CutSmart buffer (New England Biolabs). This assembly was subsequently combined with the leash precursor at $\approx 130 \mathrm{nM}$ final concentration each in $1 \times$ CutSmart buffer, along with 10 units (U) of Sbfl-HF, $10 \mathrm{U}$ of Mlul-HF, $10 \mathrm{U}$ of Nsil-HF, $5 \mathrm{U}$ of Ascl, $1000 \mathrm{U}$ of T4 DNA ligase (New England Biolabs), 1 mM ATP, and 1 mM DTT (Step a4 in Supp. Fig. 1). After overnight incubation at $25^{\circ} \mathrm{C}$ the mixture was heat inactivated at $65^{\circ} \mathrm{C}$ for $20 \mathrm{~min}$. Then, in a one pot reaction taking place at $37^{\circ} \mathrm{C}$ for $8-16 \mathrm{~h}$, the tips were nicked with $30 \mathrm{U}$ of $\mathrm{Nb}$. BvCl (New England Biolabs) and the extremities of the shanks were digested with $100 \mathrm{U}$ of Xbal and $50 \mathrm{U}$ of Sacl (New England Biolabs) (Step a5 in Supp. Fig. 1). The product was separated on a $1.5 \%$ agarose gel at $37^{\circ} \mathrm{C}$ and extracted using the NucleoSpin Gel and PCR Clean-up kit (Macherey-Nagel).

Finally, the $\approx 2.7 \mathrm{kbp}$ construct obtained above was ligated at the Sacl site to $\mathrm{a} \approx 1 \mathrm{kbp}$ dsDNA fragment multiply labeled with biotin and at the Xbal site to $a \approx 1 \mathrm{kbp}$ dsDNA fragment multiply labeled with digoxigenin (Step a6 in Supp. Fig. 1). Those labeled DNA fragments were synthesized by PCR as previously described [36, 56], in the presence of dUTP-biotin and dUTP-digoxigenin (Roche), respectively. The ligation reaction was conducted for $3 \mathrm{~h}$ at room temperature, in $10 \mu \mathrm{L}$ of $1 \times \mathrm{T} 4 \mathrm{DNA}$ ligase buffer (New England Biolabs) containing $3 \mathrm{nM}$ of construct, $10 \mathrm{nM}$ each of biotin and digoxygeninlabeled $\approx 1 \mathrm{kbp}$ fragments, and $200 \mathrm{U}$ of T4 DNA ligase. The enzyme was then inactivated and the resulting J-DNA tweezers were aliquoted and stored at $-20^{\circ} \mathrm{C}$.

J-DNA tweezers with a 690 bp leash and functionalized by small recognition molecules $B G$ and $B C$ 
Preparation was as above, except for two modifications. First, oligonucleotides $\mathrm{TS}_{1}$ and $\mathrm{TS}_{2}$ were replaced with oligonucleotides $\mathrm{TS}_{3}$ and $\mathrm{TS}_{4}$. Second, $\mathrm{Nb}$.BbvCl was omitted when performing the one pot digestion with Xbal and Sacl.

J-DNA tweezers with a $2.7 \mathrm{kbp}$ leash and functionalized by Nb.BbvCl-generated ssDNA overhangs

Preparation was similar to the one of the scaffold with the $690 \mathrm{bp}$ leash, with two variations. The $\approx 2.7 \mathrm{kbp}$ precursor of the leash was now generated from the 690_pUC18 plasmid from which the native Sacl site has been removed using the Quick Blunting kit (New England Biolabs). Digestion with the Mlul and Sbfl restriction enzymes yielded products of the same lengths than in the original protocol but we now purified the longer one. Second, higher amounts of enzyme were used to digest the product resulting from the ligation of the $2.0 \mathrm{kbp}$ branched PCR product to the $2.7 \mathrm{kbp}$ leash: $50 \mathrm{U}$ of $\mathrm{Nb} . \mathrm{BbvCl}$, $200 \mathrm{U}$ of $\mathrm{Xbal}$, and $100 \mathrm{U}$ of Sacl.

\section{Protein design and production}

Standard protocols inspired by previously published reports are described in details in Supplementary information.

\section{Assembly of the proteins at the tips of the J-DNA tweezers}

\section{General strategy}

Two strategies were used in our work. In the first one, AzF-containing FRB and FKBP12 proteins were coupled via click chemistry to DBCO-modified oligonucleotides, each complementary to a specific 9-base overhang present at one of the tips of the J-DNA tweezers. Each protein could thus be addressed to the scaffold via hybridization and ligation of its accompanying oligonucleotide to a specific tip. In the second strategy, the J-DNA tweezers were prepared with BG- and BC-functionalized tips, and SNAP-FRB and CLIP-FKBP12 covalently coupled to the tweezers simply by mixing the three components together. 
Oligonucleotides sequences ( $5^{\prime}$ to $3^{\prime}$ direction)

$\mathrm{DBCO}-\mathrm{P} 1$

XATATGAGGC

$\mathrm{DBCO}-\mathrm{P} 2$

xTGTAAGAGC

In the present notations $\mathbf{X}$ indicates DBCO-dT.

Recognition by hybridization of complementary oligonucleotides and ligation

Between 40 and $50 \mu$ g of DBCO-labelled oligonucleotides were resuspended in $5 \mu \mathrm{L}$ of water and combined with $\approx 70 \mu \mathrm{L}$ of $170 \mu \mathrm{M}$ AzF-containing protein in $50 \mathrm{mM}$ Tris- $\mathrm{HCl}, \mathrm{pH} 7.5$ buffer containing $300 \mathrm{mM} \mathrm{NaCl}$ and $10 \%$ glycerol. This equimolar mixture was incubated for $2 \mathrm{~h}$ at $25^{\circ} \mathrm{C}$ for FKBP12 derivatives or overnight at $4{ }^{\circ} \mathrm{C}$ for FRB ones (Step b1 in Supp. Fig. 1). These conditions typically yielded $50 \%$ labeling of proteins, as estimated using Cy5-DBCO fluorescent dye (Sigma-Aldrich) as a model reagent and measuring the reaction yield by gel electrophoresis. Typically, FRB A2020AZF $_{\text {and }}$ FRB $_{\text {E2052AZF }}$ were covalently labeled with $\mathrm{DBCO}-\mathrm{P} 1$ and, in consequence, were closer to the magnetic bead, whilst FKBP12 $2_{\text {MOAZF }}$ and FKBP12 $12_{\text {R13AzF }}$ were covalently labeled with DBCO-P2, situating them closer to the glass surface. Incidentally, for experiments involving homotypic scaffolds (i.e. scaffold functionalized with

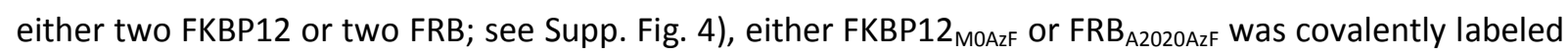
with both $\mathrm{DBCO}-\mathrm{P} 1$ and $\mathrm{DBCO}-\mathrm{P} 2$. Conjugated proteins were purified by gel filtration chromatography using a Superdex 75 Increase column (GE Healthcare) equilibrated in $50 \mathrm{mM}$ Tris- $\mathrm{HCl}, \mathrm{pH}$ 7.5, $300 \mathrm{mM}$ $\mathrm{NaCl}$, and $10 \%$ glycerol, collecting $200-250 \mu \mathrm{L}$ fractions. Concentration of oligonucleotide-protein conjugates was estimated with a DS $11 \mathrm{FX}+$ Spectrophotometer/Fluorimeter (DeNovix) using theoretical epsilon values at $280 \mathrm{~nm}\left(\varepsilon_{280}\right)$ of $32070 \mathrm{M}^{-1} \mathrm{~cm}^{-1}$ for $\mathrm{FRB}_{\mathrm{A} 2020 \mathrm{AZF}}$ and $\mathrm{FRB}_{\mathrm{E} 2052 \mathrm{AzF}}, 30580 \mathrm{M}^{-1} \mathrm{~cm}^{-1}$ for $\mathrm{Y} 2105 \mathrm{~A}$

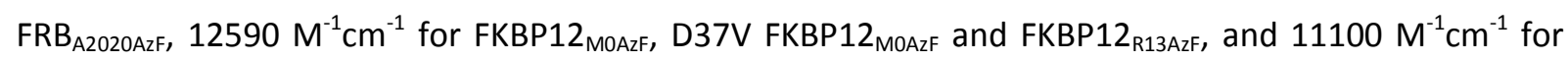
Y82F FKBP12 ${ }_{\text {MOAZF. }}$

Oligonucleotide-protein conjugates were ligated onto the $\mathrm{Nb.BbvCl-nicked} \mathrm{J-DNA} \mathrm{tweezers} \mathrm{using}$ T4 ligase (New England Biolabs) in mTOR buffer (20 mM K.HEPES, pH 7.8, $100 \mathrm{mM} \mathrm{KCl}, 5 \mathrm{mM} \mathrm{MgCl}, 0.1$ 
\% Tween-20, $0.5 \mathrm{mg} / \mathrm{mL}$ BSA, $2 \mathrm{mM}$ DTT) supplemented with $1 \mathrm{mM}$ ATP and $2 \mathrm{mM}$ DTT (Step b2 in Supp. Fig. 1). Reaction volumes of $50 \mu \mathrm{L}$ containing $20-200 \mathrm{nM}$ of each protein conjugate and $50 \mathrm{pM}$ of J-DNA were incubated for at least $4 \mathrm{~h}$ at $16^{\circ} \mathrm{C}$. Then, glycerol was added to a final concentration of $10 \%$ and assembled scaffolds were aliquoted, frozen in liquid $\mathrm{N}_{2}$, and stored at $-80^{\circ} \mathrm{C}$.

One of the above conjugation reactions between a DBCO-labelled oligonucleotide and an AzFcontaining protein, followed by purification, is sufficient for at least 100 - 150 subsequent engraftment reactions. One of the latter ligations onto the J-DNA tweezers requires as little as $1-10$ pmol of each oligonucleotide-protein conjugate. Yet, it provides enough material for at least 40 single-molecule experiments. Therefore, one conjugation reaction enables to perform at least $4000-6000$ singlemolecule experiments.

Coupling of SNAP and CLIP protein tags to small-molecule substrates BG and BC

The SNAP- and CLIP-tagged proteins respectively react with the BG and BC groups that are located at the tips of the $\mathrm{BG} / \mathrm{BC}$-containing J-DNA tweezers, forming a stable thioether covalent bond $[49,55]$. Typically, reaction volumes of $15 \mu \mathrm{L}$ containing $2 \mu \mathrm{M}$ of each fusion protein and $1 \mathrm{nM}$ of BG/BCcontaining J-DNA in mTOR buffer were incubated overnight at $25^{\circ} \mathrm{C}$ (Step b2' in Supp. Fig. 1). Reaction mixtures were then diluted 20-fold with mTOR buffer and glycerol was added to a final concentration of $10 \%$. Finally, the assembled scaffolds were aliquoted, frozen in liquid $\mathrm{N}_{2}$, and stored at $-80^{\circ} \mathrm{C}$.

The previous assembly reaction requires as little as $12 \mathrm{fmol}$ of each SNAP- and CLIP-fused protein. Yet, the obtained amount of J-DNA tweezers conjugated with proteins is sufficient for at least 250 single-molecule experiments.

\section{Single molecule experiments workflow}

\section{Reagents}

Stock solutions of rapamycin (Sigma-Aldrich) and deforolimus (MedChem Express) were prepared by dissolution at $50 \mu \mathrm{M}$ in dimethyl sulfoxide (DMSO, Sigma-Aldrich) whilst everolimus (SigmaAldrich) was supplied in acetonitrile and diluted to $100 \mu \mathrm{M}$ in the latter solvent. Single-use aliquots of stock solutions were stored at $-80^{\circ} \mathrm{C}$. 


\section{Experimental conditions}

All assays were conducted in mTOR buffer (20 mM K.HEPES, pH 7.8, $100 \mathrm{mM} \mathrm{KCl,} 5 \mathrm{mM} \mathrm{MgCl}$, $0.1 \%$ Tween-20, $0.5 \mathrm{mg} / \mathrm{mL}$ BSA, $2 \mathrm{mM}$ DTT). Rapamycin and rapalogs were used at a final concentration of $500 \mathrm{nM}$ unless stated otherwise and the concentration of dimethyl sulfoxide or acetonitrile in the mTOR buffer was always at 1 or $0.5 \% \mathrm{v} / \mathrm{v}$, respectively. For Fig. 3a, data collected at $21.7^{\circ} \mathrm{C}$ and for high forces greater than $30 \mathrm{fN}$ were obtained at $390 \mathrm{nM}$ rapamycin.

Temperature was set at $21.7^{\circ} \mathrm{C}$ unless stated otherwise.

\section{Flow cell preparation}

Flow cells for single-molecule experimentation were prepared as extensively described elsewhere [36, 56], starting from two glass coverslips (\#1, $\approx 180 \mu \mathrm{m}$ thick, Menzel-Glaser), one of them having been sandblasted to provide two 2-mm in diameter access ports. Those coverslips are spincoated first with MCC Primer 80/20 (MicroChem) and second with a polystyrene solution $(0.1 \% \mathrm{w} / \mathrm{v}$ in toluene). Then, they are assembled thanks to two thicknesses of parafilm M (75 $\mu \mathrm{m}$, Bemis North America) into which a channel $(1 \mathrm{~mm} \times 50 \mathrm{~mm})$ has been cut open. Further surface functionalization with antidigoxigenin (Roche) and passivation are achieved in the closed fluidic channel, as described [36, $56]$.

\section{J-DNA tweezers assembly}

J-DNA tweezers are attached to streptavidin-coated magnetic bead at the biotin-labeled end (i.e. via the $\approx 690$ bp long branch) and to the antidigoxigenin-treated glass surface at the dioxigeninlabeled end (i.e. via the $\approx 1330$ bp long branch).

More precisely, 1- $\mu$ m-diameter streptavidin-coated magnetic beads (MyOne Streptavidin C1, Life Technologies) were first prepared by washing $5 \mu \mathrm{L}$ of stock solution $(10 \mathrm{mg} / \mathrm{mL})$ with $200 \mu \mathrm{L}$ of mTOR buffer. After magnetic concentration and disposal of the supernatant, the particles were resuspended in $35 \mu \mathrm{L}$ of mTOR buffer. Then, $0.5-1.4 \mu \mathrm{L}$ of solution of functionalized J-DNA tweezers, at $50 \mathrm{pM}$, were added. This mixture was incubated for $2 \mathrm{~min}$ at room temperature, injected in the flow cell 
and incubated for an additional 10 min before washing away the unattached beads with mTOR buffer. After mounting of the microfluidic chamber onto the microscope stage, 30 - 70 scaffolds can typically be observed per field of view and handled with magnetic tweezers. Among them up to $30 \%$ have both tips filled with proteins.

In experiments using homotypic scaffolds (Supp. Fig. 4), J-DNA constructs were assembled under the magnetic tweezers and exposed for $15 \mathrm{~min}$ to $50 \mathrm{nM}$ rapamycin and $50 \mathrm{nM}$ of complementary protein dimer (i.e. either FRB-PEG - FRB or FKBP12-PEG ${ }_{4}-\mathrm{FKBP} 12$ ). Free components were then washed out with $4-5 \mathrm{~mL}$ of mTOR buffer, and $50 \mathrm{nM}$ of the appropriate protein dimer reintroduced into the chamber before beginning measurements.

In experiments carried out at limiting concentrations of rapamycin (Supp. Fig. 7), scaffolds were assembled under the magnetic tweezers and exposed for $15 \mathrm{~min}$ to $50 \mathrm{nM}$ rapamycin. Free rapamycin was then washed out with $4-5 \mathrm{~mL}$ of mTOR buffer before beginning measurements. From the ratio of the used volumes, the concentration of the remaining rapamycin was estimated to be lower than 250 pM (the microfluidic chamber with fillports is $\approx 20 \mu \mathrm{L}$ in volume).

\section{Analysis of pulling vector direction}

A support vector machine (SVM) model in Scikit-learn (http://scikit-learn.org/stable/about.html) was used to estimate the plane of separation between the two interacting protein faces. A SVM is a classification algorithm that identifies a hyperplane between categories and that maintains the largest distance to data points of different categories. This was leveraged to identify a plane with the largest distance to the nearest coordinate of either protein. It was observed that the normal vector to the separation plane ( $\vec{Z}$, depicted in blue in Fig. 4a) was oriented similarly to the longest rotational axis of the protein complex. This was used to identify a second vector $(\vec{X}$, depicted in grey and pointing toward the reader in Fig. 4a) that was parallel to the separation plane and closest in orientation to the secondlongest rotational axis. A third vector ( $\vec{Y}$, depicted in grey and vertical in Fig. 4a) was identified as the vector that completed the basis. 


\section{Magnetic tweezers operation}

Details on this well-known single-molecule techniques $[57,58]$ can be found in Supplementary information.

\section{Data analysis}

We used custom routines in the Xvin software suite (PicoTwist). For cycling-force experiments traces of DNA extension versus time were analyzed to determine (i) $\Delta t$, the duration of the interaction, which corresponds to the dwell-time in low-extension state at high force; (ii) $\Delta z$, the extension change upon dissociation, which corresponds to the difference in bead height before and after rupture at high force (Fig. 2). We excluded from analysis all the events for which rupture did not occur within the same force-traction cycle as the one in which binding was first observed. Indeed, they could not be reliably assessed for continuity. For presentation purposes time-traces are offset in extension so that the maximal extension is that expected from the worm-like chain model for $a \approx 2.7 \mathrm{kbp}$ DNA at the given extending force - in the case of the longer $2.7 \mathrm{kbp}$ leash of Supp. Fig. 9 we used a $\approx 4.7 \mathrm{kbp}$ contour length.

Roughly 100 - 500 events were collected from each scaffold molecule monitored. Event durations were represented in histogram form using the appropriate bin size (optimal bin sizes were typically $\approx 10 \%$ of the mean lifetime, i.e. $\approx 1 \mathrm{~s}$ bins for events with mean lifetimes of $\approx 10 \mathrm{~s}$ and $\approx 5 \mathrm{~s}$ bins for events with mean lifetimes of $\approx 50 \mathrm{~s}$ ). Error bars in histograms are equal to the square root of the number of events in that bin and represent the statistical (Poissonian) counting error (i.e. the standard deviation in counts). Histograms were fitted to single-exponential decays after removing the first bin (for which missed events may lead to undercounting) and after weighting points by error bars. For each scaffold molecule, a lifetime \pm SEM was thus obtained. SEM typically progressed as SD/(number of events) ${ }^{1 / 2}$ as expected for a stochastic process. We then averaged together lifetimes obtained on different scaffold molecules and computed the population variance (except in Supp. Fig. 2b, where enough scaffolds were collected to give a Gaussian fit to the population distribution). 


\section{Data Availability}

The data that support the plots within this paper and other findings of this study are available from the corresponding author upon reasonable request.

\section{References}

[55] A. Keppler, S. Gendreizig, T. Gronemeyer, H. Pick, H. Vogel, and K. Johnsson. A general method for the covalent labeling of fusion proteins with small molecules in vivo. Nat. Biotechnol., 21:86-89, 2003.

[56] A. Revyakin, R.H. Ebright, and T.R. Strick. Single-molecule DNA nanomanipulation: improved resolution through use of shorter DNA fragments. Nat. Methods, 2:127-138, 2005.

[57] T. R. Strick, J.F. Allemand, D. Bensimon, A. Bensimon, and V. Croquette. The elasticity of a single supercoiled DNA molecule. Science, 271:1835-1837, 1996.

[58] C. Gosse and V. Croquette. Magnetic tweezers: Micromanipulation and force measurement at the molecular level. Biophys. J., 82:3314-3329, 2002. 